\title{
Rashba spin-orbit coupling in a two-dimensional electron system under quantum Hall regime
}

\author{
R. Cangas ${ }^{\mathrm{a}}$, M.A. Hidalgo \\ Departamento de Física, Escuela Universitaria de Ingeniería Técnica Industrial, Universidad Politécnica de Madrid, Ronda de Valencia 3, 28012 Madrid, Spain \\ Departamento de Física, Universidad de Alcalá, Alcalá de Henares (Madrid), Spain
}

\begin{abstract}
In this paper, we analyze the contribution of the Rashba spin-orbit coupling in a two-dimensional electron system (2DES) of an inversion layer under quantum Hall regime as a function of the magnetic field. The study is based on a semiclassical model for the magnetoconductivities of the 2DES. This model reproduces the measurements of the Subnikov-de Haas oscillations obtained in III-V heterostructure, and shows the Rashba spin-orbit coupling at low fields. We also discuss the Rashba and Zeeman
\end{abstract}

The spin-orbit coupling effect in semiconductors takes importance in 1990 when Datta and Das proposed a spinpolarized field effect transistor [1]. The key idea of this device is that the orientation of spins of a two-dimensional electron system (2DES) confined in a narrow-gap semiconductor quantum well can be changed by the spin-orbit interaction, providing both parallel and anti-parallel orientations with respect to the spin-orbit magnetic field. The spin-polarized carrier are injected and collected by the ferromagnetic electrodes. The modulation of current is achieved by controlling the alignment of a carrier's spin with respect to the magnetization vector in the collector electrode. The gate electrode on the top of the device is used to control the spin-orbit interaction that depends on the gate electric field. Even without any external magnetic/electric field, the carriers of the 2DES can be spin polarized by the internal built-in electric field due the structure inversion asymmetry (SIA) of the heterostructure device. The first theoretical study of this effect was made by Rashba in 1960 [2]. In 1989 Das et al. obtained an evidence of spin splitting at zero magnetic field in InGaAs/ InAlAs heterostructures [3]. The SIA electric field is normal to the
2DES confined in the inversion layer or quantum well, and the spin splitting provided by this field is given by the expression [4]

$$
\Delta E_{S O}^{S I A}=2 \alpha k
$$

where $\alpha$ is a parameter that depends on the electric field asymmetry of the heterostructure, and $\vec{k}=\left(k_{x}, k_{y}\right)$ is the 2DES wave vector, $\alpha$ is also called Rashba parameter. Measured values of $\alpha$ varies between $2 \times 10^{-12}$ and $5 \times 10^{-11} \mathrm{eVm}$ for a $2 \mathrm{DES}$ confined in InGaAs/InAlAs heterostructures [5,6]. Also in MOSFET devices $\alpha$ can be tuned with the gate voltage $[1,7]$. On the other hand, zincblend semiconductors have bulk inversion asymmetry (BIA), varying their lattice potential with the crystal directions and therefore the local electric field [8]. For a 2DES confined in a $(x, y)$ plane the energy is spin-orbit splitted by this local field, giving $\Delta E_{S O}^{B I A}=\gamma\left(k_{x} k_{y}\right) k[9]$, where $\gamma$ is a parameter that depends on the material. The BIA effect is stronger than the SIA effect in the GaAs/ AlGaAs heterostructure 2DES [10], and the values measured of spin-split energy in this alloy are of the order of $20 \mu \mathrm{eV}$ at the Fermi level [11]. In quantum well and heterostructure devices made with InGaAs/InAlAs systems the SIA effect has more relevance than BIA, obtaining spin-split energies of the order of $\mathrm{meV}$ at Fermi level [5]. This has hindered spintronics research in the GaAs/AlGaAs system, which provides the highest mobility 2DES. 
In this paper, we reproduce the experimental results obtained by Nitta et al. [7], where they realized several measurements of the Subnikov-de Haas (SdH) oscillations of a 2DES, confined in an $\mathrm{In}_{0.53} \mathrm{Ga}_{0.47} \mathrm{As} / \mathrm{In}_{0.52} \mathrm{Al}_{0.48} \mathrm{As}$ heterostructure at $0.4 \mathrm{~K}$. A regular Hall bar sample was made with this structure and the 2DES is placed in the $\operatorname{In}_{0.53} \mathrm{Ga}_{0.47}$ As layer. An applied voltage $V_{G}$ on top of device induces a variation on the Rashba parameter $\alpha$ of the 2DES, which is obtained from the node positions of $\mathrm{SdH}$ oscillations. In fact, they show that a negative gate voltage increases the electric field and enhances $\alpha$. The model that we use is a simple semiclassical theory of a two-dimensional electron gas confined in a well, under the application of external electric and magnetic fields, and with the presence of Rashba spin-orbit coupling (SIA effect). In this theory, we assume that variations in the external magnetic field produces a fluctuation of the electron density, keeping constant the chemical potential, which is fixed by the environment [12]. In all 2DES confined in heterostructures there is a reservoir of electrons surrounding the system that provides electrons. Then, necessarily the chemical potential is established by the whole 3D structure. A similar scenario was intended at the beginning of the discovery of the quantum Hall effect [13], although considering a capture mechanism of electrons by the impurities.

At zero external magnetic fields, we consider the 2DES like a two-dimensional non-interacting electron gas under the effective mass approximation, perturbed by impurities, defects and the spin-orbit coupling. Then, the energy of each electron can be approached as $E(k)=\hbar^{2} k^{2} / 2 m+U \pm \Delta E_{S O} / 2$, where $m$ is the electron effective mass, $U$ takes into account the electrostatic interaction with impurities and defects, and $\Delta E_{S O}$ is the spin-orbit split energy caused by the SIA effect. Hence, each energy level is split into two levels spaced in energy by a factor $\Delta E_{S O}$. Then the whole 2DES can be studied like two 2DES with different spin orientations (parallel and anti-parallel to the spin-orbit magnetic field). On the other hand, when an external magnetic field $B$ is applied normal to the 2DES, and assuming no spin-orbit coupling effects, the energy of the system is discretized in Landau Levels (LL), with values $E_{N_{I I}}^{ \pm}=\left(N_{N_{I I}}+1 / 2\right) h \omega \pm 1 / 2 g \mu B$, where the last term (Zeeman term) correspond to spin $\uparrow \downarrow$ orientations, $N_{L L}=0,1,2,3, \ldots, g$ is the effective $g$-factor, $\omega=e B / m$ the cyclotron frequency and $\mu$ the Bohr magneton. Measured values of $g$-factor varies from -0.44 [14] in GaAs to -30 [15] in InAs alloys, and depends on the carrier concentration [16] (we assume in this work a constant value of $g$ factor).

In a 2DES confined in a heterostructure device, taking into account the SIA and Zeeman effects, the energy of carriers is obtained by the expression [4]

$E_{N_{L}}^{\varsigma}=\hbar \omega\left[N_{L}+s \frac{1}{2} \sqrt{\left(1-|g| \frac{m}{2 m_{0}}\right)^{2}+\frac{\gamma}{B} N_{L}}\right]$

with $s= \pm 1$ for $N_{L}=1,2,3, \ldots, s=+1$ for $N_{L}=0$ (the index $s$ refers to spin $\uparrow \downarrow$ orientations), $\gamma=8 \alpha^{2} m^{2} / h^{3} e$ and $m_{0}$ are the rest electron mass. Fig. 1 shows a fan of energy levels of the 2DES with data obtained from Table 1 for $V_{G}=0.3 \mathrm{~V}$ (see below). In the figure a crossing of spin-up $\left(E_{N_{L}}^{+}\right)$and spin-down $\left(E_{N_{L}^{\prime}}^{-}, N_{L} \neq N_{L}^{\prime}\right)$ energy levels is appreciated for some values of the magnetic field. We will show the consequences of this behaviour in the magnetoconductivity properties of the 2DES.

It is well known that the density of states in the 2DES at zero field is $D_{0}=m / 2 \pi \hbar^{2}$, i.e. the states are uniformly distributed in energies (in this equation we have not taken into account the spin degeneration). But when a magnetic field is applied the energy states are given by Eq. (2). In this approximation, we assume that each level is degenerated in $e B / h$, and that the density of states has a Gaussian shape. Then, we can write the following

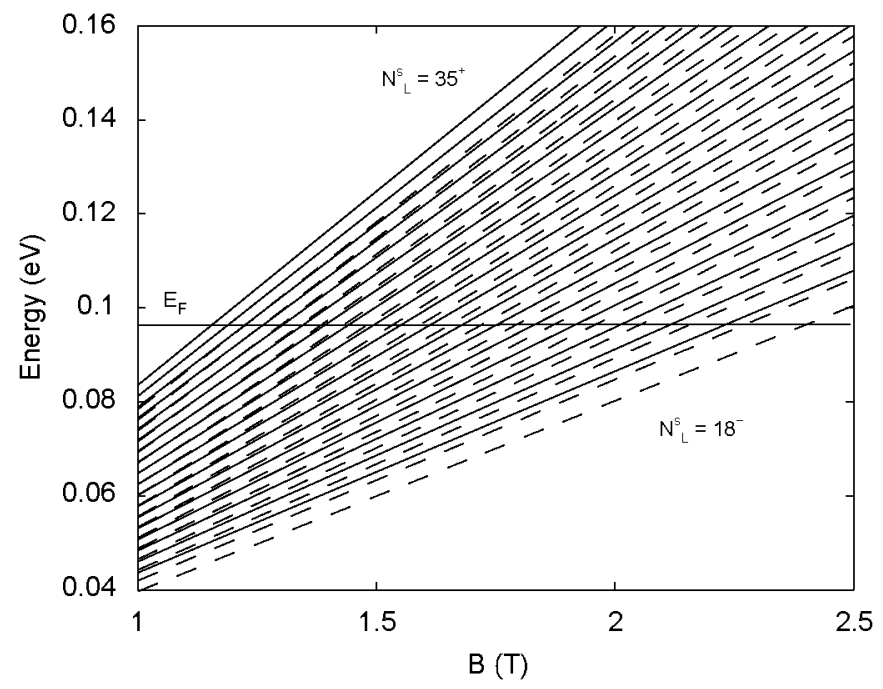

Fig. 1. Plot of the energy levels (from $N_{L}=18$ to 35 ) of the 2DES system with the data of Table 1 for $V_{\mathrm{G}}=0.3 \mathrm{~V}$. Dash line corresponds to $E_{N_{L}}^{-}$levels and solid line to $E_{N_{I}}^{+}$levels. It shows the crossing of spin-up and spin-down levels when $B$ decreases.

Table 1

Parameters used to compute the magnetoresistance of the 2DES confined in a $\operatorname{In}_{0.53} \mathrm{Ga}_{0.47}$ As layer of the device described in Ref. [7].

\begin{tabular}{llll}
\hline$V_{G}$ (volts) & $n\left(\mathrm{~m}^{-2}\right)$ & $\alpha(\mathrm{eVm})$ & $\Gamma_{N_{L_{S}}}$ \\
\hline 0.3 & $2.0 \times 10^{16}$ & $7.2 \times 10^{-12}$ & $0.010 E_{F}+\sqrt{\left(2 \hbar^{2} / \pi\right)(\omega / \tau)}$ \\
0.0 & $1.9 \times 10^{16}$ & $7.7 \times 10^{-12}$ & $0.014 E_{F}+1.4 \sqrt{\left(2 \hbar^{2} / \pi\right)(\omega / \tau)}$ \\
-0.3 & $1.8 \times 10^{16}$ & $8.3 \times 10^{-12}$ & $0.016 E_{F}+1.6 \sqrt{\left(2 \hbar^{2} / \pi\right)(\omega / \tau)}$ \\
\hline
\end{tabular}

The effective mass of the carriers is $m=0.05 m_{0}$, the effective $g$-factor is -4 . The relaxation time is $\tau=1.0 \times 10^{-12} \mathrm{~s}$, obtained from the measures made by Burgt et al. [20].

ad hoc expression:

$D(E)_{s}=\frac{e B}{h} \sum_{s} \sum_{N_{L}}\left[\frac{\pi}{2} \Gamma_{N_{L} s}^{2}\right]^{-1 / 2} \exp \left\{-2 \frac{\left(E-E_{N_{L}}^{s}\right)^{2}}{\Gamma_{N_{L} s}^{2}}\right\}$

where $\Gamma_{N_{L} S}$ is the width of the $E_{N_{L}}^{s}$ level. The level broadening is strongly dependent on the range of scattering potentials [16]. For short-range scatters $\left(d<l / \sqrt{\left(2 N_{L}+1\right)}\right.$, where $d$ is the order of the range and $l=\sqrt{\hbar} / e B$ the magnetic length) $\Gamma_{N_{L} S}^{2}$ depends on the strength of the magnetic field. The broadening due to long-range potentials is proportional to fluctuations of the local potential energy $(V(r)-\langle V(r)\rangle)^{2}$, and can be considered negligible for shortrange potentials in $\delta$-doped samples where the impurities are far from the 2DES. Then, we use the expression

$\Gamma_{N_{L} s}=\Gamma_{0}+\kappa \sqrt{\left(2 \hbar^{2} / \pi\right)(\omega / \tau)}$

where $\Gamma_{0}$ and $\kappa$ are fitting parameters, and $\tau$ is the relaxation time without applied magnetic field. Eq. (3) involves two 2DES with two different spin states. Fig. 2a and b shows the density of states of a $2 \mathrm{DES}$ for the data given in Table 1 for $V_{G}=0.3 \mathrm{~V}$ at 1.2 and $1.8 \mathrm{~T}$, respectively. When the applied magnetic field increases, the energy levels $E_{N_{L}}^{S}$ move to the Fermi level $\left(E_{F}\right)$, and the conduction occurs when each level crosses $E_{F}$, providing a modulated oscillation in the magnetoconductivity and magnetoresistivity at quantum Hall regime (beating pattern of SdH oscillations, see Fig. 3) [17]. The beating pattern arises from the existence of two kinds of carriers, and the sum of their concentrations at Fermi level. The nodes of the oscillations occur in the region of magnetic field in which there is no coincidence of energy levels at Fermi 


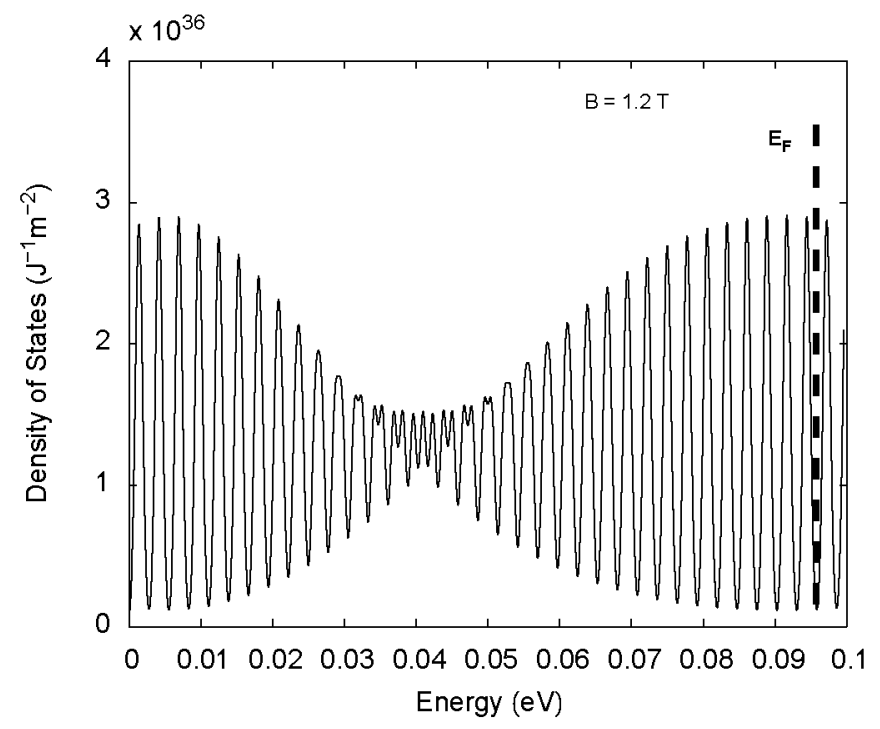

b

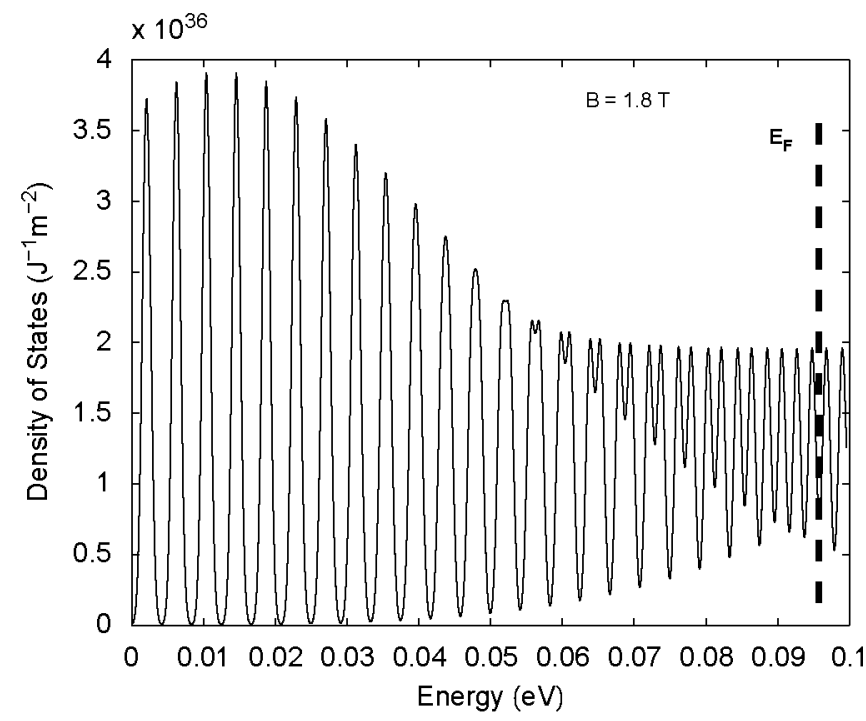

Fig. 2. The 2DES density of states with data from Table 1 for $V_{G}=0.3 \mathrm{~V}$. (a) At $B=1.2 \mathrm{~T}$, where there is coincidence of adjoining levels at energies close to $E_{F}$ (b) at $B=1.8 \mathrm{~T}$, where all levels are spin resolved at energies near $E_{F}$ and hence there is no coincidence of levels.

energy. This condition is given by $\left(E_{N_{L}}^{+}+E_{N_{L}-1}^{+}\right) / 2=E_{N_{L n C}}^{-} \approx E_{F}$, where $N_{L n C}=N_{L}+1, N_{L}+2, \ldots$. . The first node occurs close to $1.8 \mathrm{~T}$, when the levels $N_{L} \approx 22-23$ cross the Fermi level and $N_{L n C}=N_{L}+1$. The second node occurs at $0.95 \mathrm{~T}$ with $N_{L} \approx 43$ and $N_{L n C}=N_{L}+2$. High-order nodes occur for $N_{I n C} \geqslant N_{L}+3$ and $B<0.95$ T. On the other hand, when $E_{N_{L}}^{+}=E_{N_{L C}}^{-}$, with $N_{L C}=N_{L}+1, N_{L}+2, \ldots$, a coincidence of levels appears. At Fermi level, one coincidence occurs in a region of magnetic field near to $1.2-1.3 \mathrm{~T}$ for $N_{L} \approx 31-33$ (with $N_{L C}=N_{L}+2$ ), and in this region the magnetoconductivity oscillations have maximum local values. In Fig. 2a there is coincidence of levels near $E_{F}$ but not in Fig. 2b. Also exists a competition between Rashba and Zeeman effects which occurs by the coincidence of levels $E_{N_{L}}^{+}$and $E_{N_{L}+1}^{-}$. If we compare Eq. (2) with the conventional spin-up and spin-down energy states associated with $N_{L}$ LL number, this correspond to $E_{N_{i}}^{+}$and $E_{N_{i}+1}^{-}$states, i.e. $\Delta E_{s p i n}=$ $\left|E_{N_{i}}^{+}-E_{N_{L}+1}^{-}\right|$[18]. In the absence of Rashba effect, Eq. (2) reproduces well known LL energy spectrum. In the limit of large

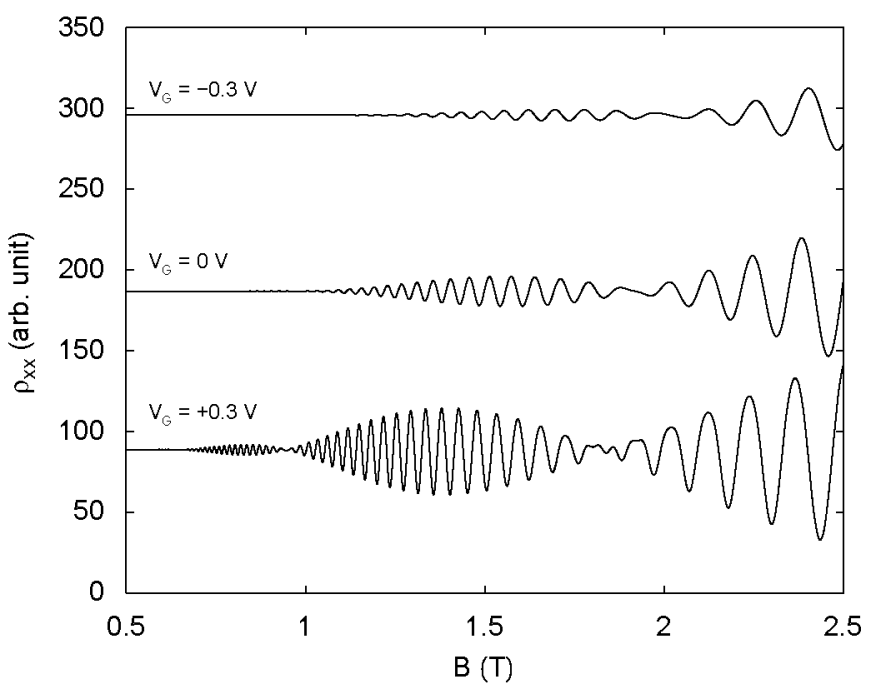

Fig. 3. SdH oscillations of a $2 \mathrm{DES}$ confined in the $\operatorname{In}_{0.53} \mathrm{Ga}_{0.47}$ As layer of a heterostructure computed with data obtained in Ref. [7] and using the parameters given in Table 1 . This model reproduces the experimental values in which a variation of Rashba parameter is observed with the gate voltage $V_{G}$ applied to the system.

magnetic fields the Zeeman term dominates the spin splitting, obtaining $\Delta E_{\text {spin }}=g \mu B$. In the opposite limit, when $B \rightarrow 0$, $\Delta E_{\text {spin }}=2 \alpha k_{F}$ is obtained, where $k_{F}=\sqrt{2 \pi n}$ is the Fermi wave vector and $n$ the equilibrium carrier concentration. The condition of coincidence is $E_{N_{L}}^{+}=E_{N_{L}+1}^{-}$, and is governed by the equation

$\sqrt{\left(1-|g| \frac{m}{2 m_{0}}\right)^{2}+\frac{\gamma}{B} N_{L}}+\sqrt{\left(1-|g| \frac{m}{2 m_{0}}\right)^{2}+\frac{\gamma}{B}\left(N_{L}+1\right)}=2$

With the data of Table 1 for $V_{G}=0.3 \mathrm{~V}$, this occurs at $4.85 \mathrm{~T}$ and $N_{L} \approx 7-8$.

The magnetoconductivities are obtained relating the carrier current density with the applied electric and magnetic fields. The general expression is

$\vec{j}(\vec{r}, t)=\vec{j}_{+}+\vec{j}_{-}=e \sum_{s} \int_{-\infty}^{\infty} \vec{v}_{s} f(E) D(E)_{s} d E$

where $\vec{v}$ is the carrier velocity, $E$ the electron energy and $f$ the distribution function perturbed by the electric and magnetic fields [19]. Hence, we are assuming two currents with different spins (parallel and anti-parallel to magnetic field). To compute the magnetoconductivity, we use the semiclassical theory, using the Eq. (3) as density of states. Taking into account the linear relationship $\vec{j}=[\sigma] \vec{E}$, where $\vec{E}$ is the applied electric field and $[\sigma]$ the magnetoconductivity tensor, we obtain

$\sigma_{x x}=\sigma_{y y}=\frac{e^{2} N \tau}{m} \frac{1}{1+(\omega \tau)^{2}}$

$\sigma_{x y}=-\sigma_{y x}=\frac{e^{2} n \tau}{m} \frac{\omega \tau}{1+(\omega \tau)^{2}}$

$n$ is the whole equilibrium carrier concentration and $N$ the carried concentration at the Fermi level, given by the expressions

$$
\begin{aligned}
& n=n_{+}+n_{-}=\sum_{s} \int_{-\infty}^{\infty} f_{0}(E) D(E)_{s} d E \\
& N=N_{+}+N_{-}=\sum_{s} \int_{-\infty}^{\infty} D(E)_{s} E\left(-\frac{\partial f_{0}}{\partial E}\right) d E
\end{aligned}
$$




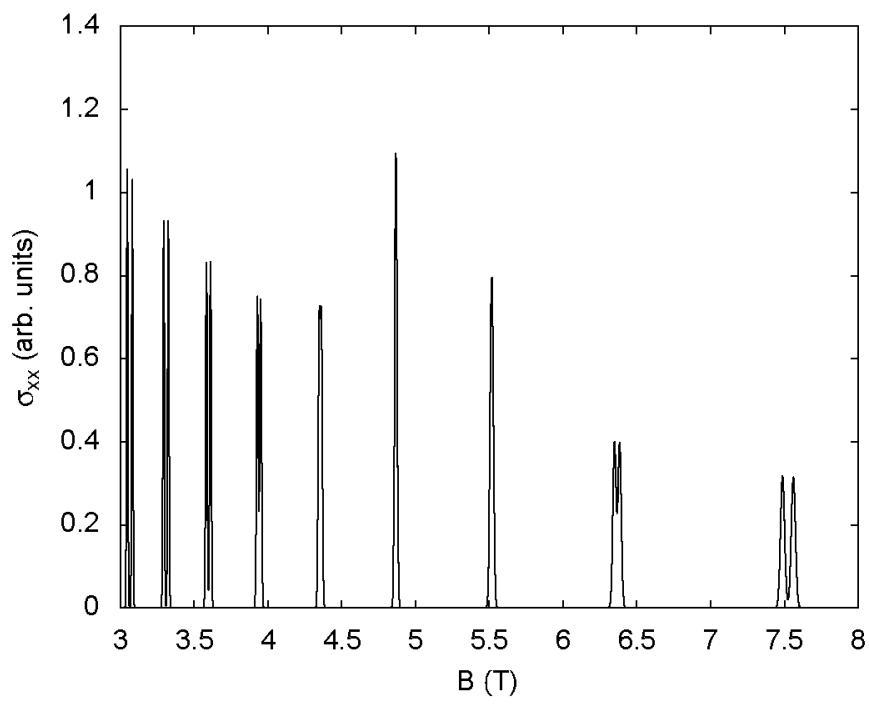

Fig. 4. Computed magnetoconductivity for a clean 2DES. The higher value of the magnetoconductivity near $4.85 \mathrm{~T}$ is due to the competition of Rashba and Zeeman effects. (The data used correspond to $V_{G}=0.3 \mathrm{~V}$ and $0.1 \Gamma_{N_{L S}}$ from Table 1.)

From Eqs. (7a) and (7b) the magnetoresistivity tensor is obtained by the relationship $[\rho]=[\sigma]^{-1}$, obtaining

$\rho_{x x}=\rho_{y y}=\sigma_{x x} /\left(\sigma_{x x}^{2}+\sigma_{x y}^{2}\right)$

$\rho_{x y}=-\rho_{y x}=-\sigma_{x y} /\left(\sigma_{x x}^{2}+\sigma_{x y}^{2}\right)$

Fig. 3 shows the computed values of magnetoresistivity from Eq. (9a) of a $2 D E S$ confined in the $\operatorname{In}_{0.53} G a_{0.47}$ As heterostructure described in Ref. [7]. From these results, we determine the data showed in Table 1 for three gate voltages, obtaining a good agreement with the experimental data measured. In fact, the model also reproduces the experimental results of the variation of the Rashba parameter with the applied gate voltage.

At $V_{G}=0.3$ the maximun of local oscillations occur at a value of magnetic field near to $1.4 \mathrm{~T}$ instead in the range $1.2-1.3 \mathrm{~T}$ as we expected if the coincidence condition $E_{N_{L}}^{+}=E_{N_{L}+2}^{-}=E_{F}$ is applied. This is a consequence of the overlapping of adjoining levels and the increase of the density of states as the magnetic field grows.
On the other hand, as we have seen before, the Rashba-Zeeman competition at Fermi levels occurs near to $4.85 \mathrm{~T}$. Shen et al. [21] deduced the appearance of a resonance in the spin Hall conductance at values of magnetic field where exists the coincidence $E_{N_{L}}^{+}=E_{N_{L}+1}^{-}$being Eq. (5) the resonant condition at Fermi level. The effect that we deduced is an increase in the charge magnetoconductivity at this value of the field, and it could be observed only in clean samples. Fig. 4 shows a plot of $\sigma_{x x}$ computed using a Gaussian width of a tenth of $\Gamma_{N_{i s}}$.

In summary, we have studied the transport properties of a 2DES with a Rashba spin-orbit coupling in a perpendicular magnetic field, where we have assumed the system composed by two kinds of carriers (electrons with parallel and antiparallel spins). We analyze the effects on the conduction of the energy levels crossing. When this occurs at Fermi energy the charge magnetoconductance increases. Also the model shows the Rashba-Zeeman competition, and predices the behaviour of $\sigma_{x x}$ at this point. The good agreement with the experimental data obtained in Ref. [7] allows the use of this simple theory to model spintronic devices.

\section{References}

[1] S. Datta, et al., Appl. Phys. Lett. 56 (1990) 665

[2] E.I. Rashba, Fiz. Tverd. Tela (Leningrad) 2 (1960) 1224; E.I. Rashba, Sov. Phys. Solid State 2 (1960) 1109.

[3] B. Das, et al., Phys. Rev. B 39 (1989) 1411.

[4] Y.A. Bychkov, et al., J. Phys. C 17 (1984) 6039.

[5] D. Grundle, Phys. Rev. Lett. 84 (2000) 6074.

[6] C.L. Yang, et al., Phys. Rev. Lett. 96 (2006) 186605.

[7] J. Nitta, et al., Phys. Rev. Lett. 78 (7) (1997) 1335

[8] D. Dresselhaus, Phys. Rev. 100 (2) (1955) 580.

[9] P.D. Dresselhaus, et al., Phys. Rev. Lett. 68 (1992) 106

[10] E.A. de Andrada e Silva, et al., Phys. Rev. B 50 (1994) 8523.

[11] R.G. Mani, et al., Phys. Rev. B 69 (2004) 193304

[12] M.A. Hidalgo, Microelectron. Eng. 43-44 (1998) 453.

[13] A.H. MacDonald, Quantum Hall Effect: A Perspective, Ed. Jaca Book spa. Milano 1989.

[14] C. Weisbuch, et al., Phys. Rev. B 15 (2) (1977) 816

[15] V.Ya. Aleshkin, et al., Semiconductors 42 (7) (2008) 828

[16] T. Ando, et al., Rev. Mod. Phys. 54 (1982) 437.

[17] F.F. Fang, P.J. Stiles, Phys. Rev. 174 (1978) 823.

[18] R. Winkler, Spin-Orbit Coupling Effects in Two-Dimensional Electron and Hole Systems, STMP 191, Springer, ISBN 3-540-01187-0, 2003, p 69.

[19] B.M. Askerov, Electron transport phenomena in semiconductors, World Scientific Publishing Co., 1994

[20] Burgt, et al., Phys. Rev. B 52 (16) (1995) 12218

[21] A. Shen, et al., Phys. Rev. Lett. 92 (25) (2004) 256603. 